\title{
Association of Glycosylated hemoglobin with Radiological severity, Systemic inflammation and Hypercoagulability in COVID -19 patients in a hospital from Central India: A Monocentric Retrospective Observational study
}

\author{
Prakhar Gupta 1 , Tasha Samuel ${ }^{2}$, Ankitkumar Halani ${ }^{3}$, Bhupendra K Ratre ${ }^{4}$ \\ ${ }^{1}$ Assistant Professor, ${ }^{2,3}$ PG Resident, ${ }^{4}$ Professor, Department of General Medicine, L. N. Medical College and \\ J.K. Hospital, Bhopal, MP, India
}

A B S T R A C T

Background: COVID-19 is currently the most common cause of morbidity and mortality worldwide. Diabetes is also one of the most common causes of morbidity and associated complications. Identification of prognostic markers like glycated hemoglobin can help early categorization of patients and their better management. Aims and Objectives: To determine if Glycated Hemoglobin (HbA1c) levels are associated with radiological severity of COVID -19 disease, thereby predict prognosis in COVID -19 patients. Its association with D dimer and IL-6 was also evaluated to assess hypercoagulability and systemic inflammation in these patients. Materials and Methods: A monocentric retrospective observational study was conducted during admission period from August 1, 2020- October 31, 2020. HbA1c levels of 188 patients who tested positive for COVID-19 in a dedicated COVID-19 tertiary care center (L.N. Medical college and J.K. Hospital, Bhopal) were determined. They were divided into 2 groups: A (HbA1c level $\leq 6.5 \%$ ), B ( $\mathrm{HbA} 1 \mathrm{c}$ level $>6.5 \%)$. Severity of disease in terms of lung involvement in $\mathrm{HRCT}$ Chest was recorded. Their $\mathrm{SpO} 2$ and oxygen requirement at admission was also recorded. Among laboratory investigations, D dimer, IL6 Levels were obtained. Results: Out of 188 COVID-19 positive patients included in our study, there were 132 men, 56 women. Median age of patients was 66 years. Mean HbA1c level was $7.2 \%$. Of these, 87 had normal HBA $1 \mathrm{C}$ and 101 had raised HBA $1 \mathrm{C}>6.5 \%$. Oxygen was required in 58 patients with raised $\mathrm{HbA} 1 \mathrm{c}$ ( $\mathrm{RR}$ of 2.782, p value $<0.0001$ ), of which 42 patients had severe lung involvement ( $R R$ of 2.498 , p value $=0.0003) .20(22.9 \%$ ) patients with normal $\mathrm{HbA} 1 \mathrm{c}$ required oxygen on admission, out of which $11(12.6 \%)$ patients had severe lung involvement. Those who had higher $\mathrm{HbA} 1 \mathrm{c}$ levels, had higher chances of severe lung involvement $(>50 \%$ ) in radiological evaluation (RR 2.783, p-value 0.0003 , Positive Likelihood ratio: $2.78,95 \% \mathrm{Cl}: 1.60$ to $4.83,85 \%$ specificity).Significant increase in D dimer was seen in $77(76.2 \%)$ patients with raised $\mathrm{HbA} 1 \mathrm{c}$ compared to 40 patients (45.9\%) with normal HbA1c(RR-1.6582, p-value 0.0001). No significant relation between $\mathrm{HbA} 1 \mathrm{c}$ levels with IL-6 was found. Conclusion: Patients with raised HBA1C contracting COVID-19 have more severe inflammation and greater lung involvement, higher chances of oxygen requirement and are associated with higher risk of hypercoagulability. HbA1C will not only help identify hyperglycemia but also help assess severity of disease (both clinically and radiologically), systemic inflammation, hypercoagulability, thereby helps in predicting prognosis, managing patients and hasten recovery.

Key words: HbA1c; COVID-19; hypercoagulability; systemic inflammation; SpO2; D dimer; IL6

\section{Access this article online}

Website:

http://nepjol.info/index.php/AJMS DOI: 10.3126/ajms.v12i6.33574

E-ISSN: 2091-0576

P-ISSN: $2467-9100$

Copyright (c) 2021 Asian Journal of Medical Sciences

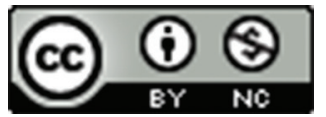

This work is licensed under a Creative Commons Attribution-NonCommercial 4.0 International License. 


\section{INTRODUCTION}

COVID-19 disease caused by (SARS-CoV-2) virus that damages lung and immune system, ${ }^{1}$ has resulted in large number of deaths worldwide. WHO declared COVID-19 outbreak a pandemic on March 11, 2020. ${ }^{2,3}$

Diabetes is leading cause of morbidity all over the world with high prevalence of $7.3 \%$ in India. ${ }^{4}$ It predisposes large proportion of population to immune dysfunction, raised inflammatory factors that causes epithelial dysfunction of pulmonary cilia, increased vascular permeability, epithelial damage and alveolar collapse, making them susceptible to various infections. ${ }^{4,5}$ Together with COVID-19, it leads to more severe inflammation, hypercoagulability, even lower oxygenation, ultimately associated with higher mortality. ${ }^{1,6}$

Angiotensin-converting enzyme 2 (ACE2) receptor, responsible for SARS coronavirus cellular invasion, favored by high and aberrantly glycated ACE2, (in pancreatic $\beta$-cells) in uncontrolled hyperglycemia, leads to higher severity in COVID-19. Virus binds to receptor releasing large amounts of inflammatory mediators, downregulation and shedding of virus-induced ACE2, thereby damaging $\beta$ cells of pancreas, eventually causes acute loss of insulin secretory capacity, thereby hyperglycemia. ${ }^{7.8}$

Hyperglycemia increases secretion of IL-6 (major proinflammatory cytokine involved in "cytokine storm"Characteristic feature of severe COVID-19 infection) and blood coagulation via imbalance between clotting factors and fibrinolysis. Increased haem breakdown produces carbon monoxide (having higher binding affinity with hemoglobin compared to oxygen) resulting in hypoxia ${ }^{9}$ which may promote intra-vessel coagulation during COVID-19 infection ${ }^{7}$ leading to significantly increased occurrence of deep vein thrombosis, pulmonary emboli and acute coronary syndrome.

Studies have shown that Poorly-controlled diabetes / stress hyperglycemia (blood glucose $>180 \mathrm{mg} / \mathrm{dl}$ ) has significantly higher risk of severe COVID-19. ${ }^{10}$ This is a retrospective study aimed to assess if glycated hemoglobin, in addition to identification of hyperglycemia, can help assess severity of disease (comparing radiological involvement and oxygen requirement) and predict prognosis in COVID -19 patients. Its association with D dimer and IL6 was evaluated to assess hypercoagulability and systemic inflammation in these patients.

\section{MATERIALS AND METHODS}

Study participants and their evaluation

It was a monocentric retrospective observational study conducted on 190 patients who tested positive for
COVID-19 in a dedicated COVID-19 tertiary care facility in Central India (L.N. Medical college and J.K. Hospital, Bhopal), during admission period from August 1, 2020 up to October 31,2020. HbA1c levels (calculating average blood glucose level in past 3 months ${ }^{1}$ ) of all these patients were obtained from records. 2 patients who had myelosuppression after leukemia chemotherapy were excluded from study due to effect on HbA1c level. After exclusion,188 patients were finally included.

\section{Inclusion criteria}

1. Those who were tested COVID -19 positive by gold standard method of molecular identification of SARSCov 2 using nucleic acid amplification test i.e.-PCR ${ }^{11}$ during admission period from August 1, 2020 up to October 31,2020,

2. Study included both patients: who were known diabetic and those who were newly diagnosed.

\section{Exclusion criteria}

- $\quad$ Age $<18$ yrs.,

- History of venous thromboembolism, patient already on anticoagulants

- Factors that affect HbA1c levels:

a. Myelosuppression after leukemia chemotherapy b. Anemia

COVID-19 patients were divided into 2 groups: A, B. Patients in group $\mathrm{A}$ had $\mathrm{HbA1} \leq 6.5$; group $\mathrm{B}$ had $\mathrm{HbA} 1 \mathrm{c}>6.5$. There were 87 patients in group A, 101 patients in group B. Disease severity was classified based on CT severity scoring, which was defined as sum of individual ground glass opacities scored in 20 lung segment regions, ranging from $0-40$ points. Optimal inflammation load score threshold for identifying severe patients was 19.5. ${ }^{12}$ Their SpO2 and oxygen requirement at admission was recorded. Of all laboratory examination results, D dimer, IL6 Levels were considered in this study. Standard lab values of markers were taken to be: $\operatorname{HbA} 1 \mathrm{C}\left(<6.5 \% 0^{13}\right)$, D-dimer $(<500 \mathrm{ng} / \mathrm{ml})^{14 *}$, IL6 $(<4.40 \mathrm{pg} / \mathrm{ml}) .{ }^{15^{*}}$

\section{Statistical analyses}

Data was summarized by using descriptive statistics. P value of $<0.05$ was considered to be statistically significant. Relative risks of $\mathrm{HbA1c}$ levels were calculated for development of severe HRCT involvement and oxygen requirement comparing those who had elevated levels of glycated haemoglobin against those who did not. Patients were divided into 4 groups based on oxygen requirement and severity of lung involvement. Average HbA1c levels for all 4 groups were also calculated to assess if groups with elevated $\mathrm{HbA} 1 \mathrm{c}$ levels had higher mean lung involvement (Figure. 1). Pearson correlation coefficients were calculated for raised glycated haemoglobin (HbA1c) and lung involvement in HRCT chest to assess the strength of association. ROC analysis was done wherever applicable. 


\section{RESULTS}

Out of 188 patients tested positive for COVID-19, there were 132 men, 56 women. Mean age of patients was 53 years, eldest being 88 yrs. old and youngest18years old. A total of 80 patients were previously diagnosed with diabetes as comorbidity. The $\mathrm{SpO}_{2}$ without oxygen inhalation at the time of admission ranged from $80 \%$ to $99 \%$. The mean HbA1c level was 7.2 (median 6.6), range 4.3-13.5\%. Out of 188 patients, 87 had normal HBA1C and 101 had raised $\mathrm{HbA1c}(>6.5 \%)$. In the subset with normal HbA1c levels, the average $\mathrm{HbA} 1 \mathrm{c}$ was $5.7 \%$ and those who had elevated levels had average HbA1c of $8.4 \%$.

A total of 101 patients had raised HbA1c levelson admission, out them 58 (57.4\%) required oxygen during the course of treatment. Results suggested significant association with raised $\mathrm{HbA} 1 \mathrm{c}$ levels and requirement of oxygen therapy (RR 2.498, p-value $<0.0001)$. Patient subset with raised HbA1c levels also showed higher chances of severe lung involvement in HRCT evaluation (RR 2.783, $\mathrm{p}$-value 0.0003$)$. The association was found to be statistically significant. Positive Likelihood ratio (raised HbA1c with $>50 \%$ lung involvement) was found to be $2.78(95 \%$ CI: 1.60 to 4.83 ) with $85 \%$ specificity and $76 \%$ positive predictive value $(95 \%$ CI $65 \%$ to $85 \%)$. A total of 127 patients had

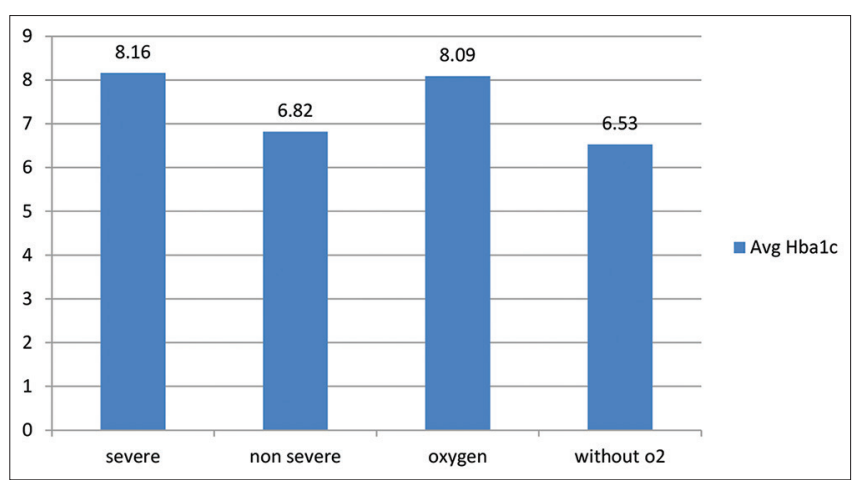

Figure 1: Average HbA1c in 4 different groups: a. severe category, b. non-severe category, c. those requiring oxygen $\mathrm{d}$. those who did not require oxygen

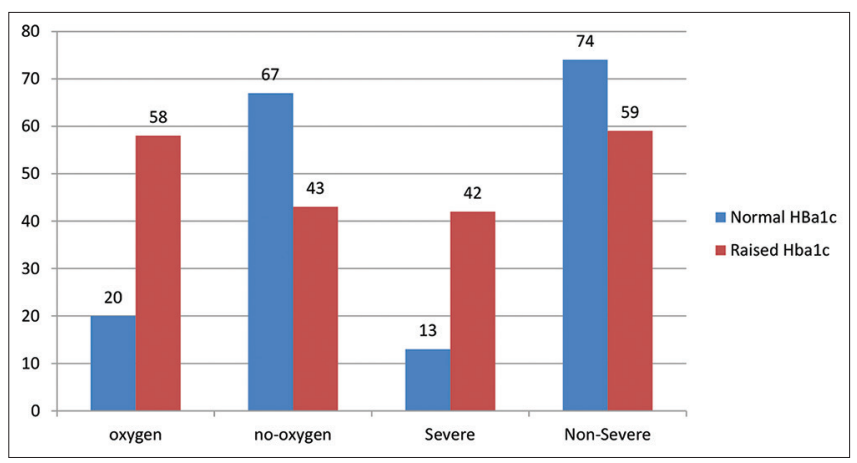

Figure 2: Comparison of $\mathrm{HbA} 1 \mathrm{c}$ levels with oxygen requirement and severity of lung involvement (CT Score>19.5\%)
CT involvement less than $50 \%$ and out them 28 patients required oxygen. Raised HbA1c showed a weak association (RR -1.839, p-value 0.0763) in this subset of patients with

\begin{tabular}{lc}
$\begin{array}{l}\text { Table 1: Correlation coefficients between HbA1c } \\
\text { levels and biomarkers }\end{array}$ \\
\hline Correlation & Correlation coefficients \\
\hline HbA1c with HRCT & 0.355515 \\
HbA1c with D-dimer & 0.110649 \\
HbA1c with IL-6 & -0.02669 \\
D-dimer with HRCT severity & 0.224216 \\
IL-6 with HRCTseverity & 0.105317 \\
\hline
\end{tabular}

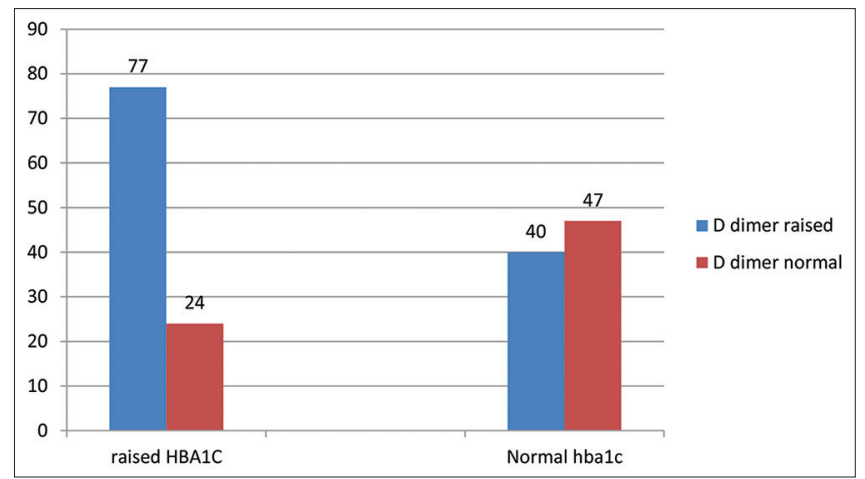

Figure 3: Comparison of $\mathrm{HbA}_{1 \mathrm{c}}$ levels with $\mathrm{D}$ dimer levels in COVID-19 patient

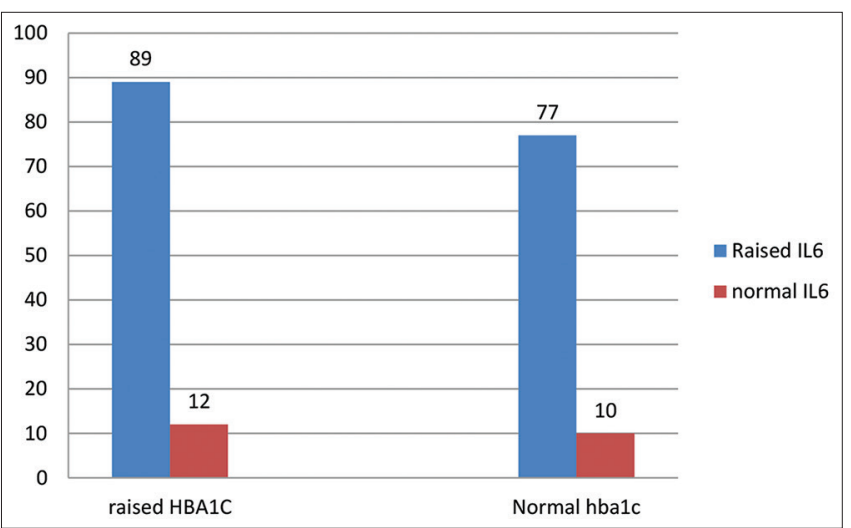

Figure 4: Comparison of $\mathrm{HbA}_{1 \mathrm{c}}$ levels with IL 6 levels in COVID-19 patient

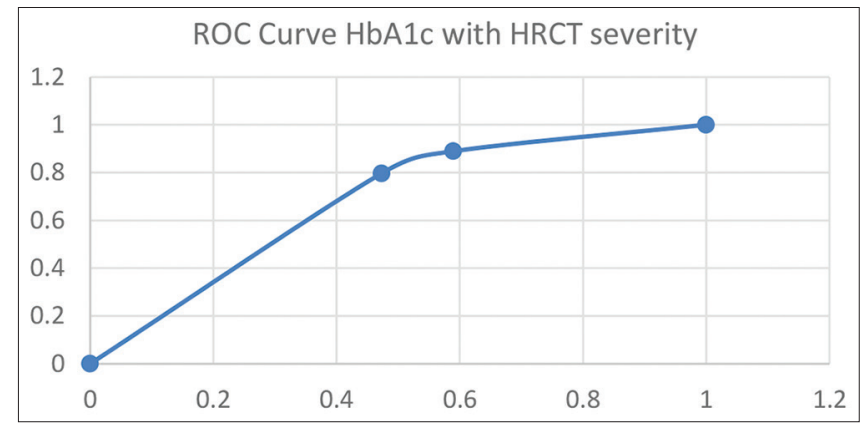

Figure 5: ROC Curve between $\mathrm{HbA}_{1}$ levels (cut-offs were taken to be $<6,6-6.5$ and $>6.5 \%$ ) and HRCT chest severity ( $<50 \%$ involvement and $>=50 \%$ lung involvement)(Area under curve: 0.890755 ) 
requirement of oxygen during treatment. Sixty-one patients had severe lung involvement on HRCT evaluation and those who had raised HbA1c showed a significant association with requirement of oxygen during the course of treatment (RR- 1.907, p-value 0.0067) (Figure 2).

Raised HbA1c levels also showed significant association with chances of raised d-dimer levels (RR-1.6582, $\mathrm{p}$-value 0.0001$)$ thereby suggesting higher chances of hypercoagulability (Figure 3). No significant association was found between IL6 levels and uncontrolled HbA1c levels (RR- 0.9956, p-value 0.9343) (Figure 4). Pearson correlation coefficients between $\mathrm{HbA} 1 \mathrm{c}$ values and HRCT chest lung involvement, D-dimer and IL-6 levels were also determined to assess the strength of association (Table 1).

HbA1c levels showed positive correlation with HRCT lung involvement and d-dimer while it showed negative correlation with IL-6 levels. ROC Curve between HbA1c levels and HRCT chest severity was also determined (Figure 5).

\section{DISCUSSION}

The purpose of this study was to determine association between uncontrolled $\mathrm{HbA} 1 \mathrm{c}$ levels, radiological and clinical severity in covid-19 patients. Diabetes together with COVID-19, leads to more severe inflammation, hypercoagulability, even lower oxygenation, ultimately associated with higher morbidity/mortality. ${ }^{1,6}$ Aberrantly glycated ACE2 receptors along with abnormal inflammatory response lead to higher severity in COVID-19. ${ }^{7.8}$ Hyperglycemia increases secretion of IL- 6 and causes imbalance in the coagulation cascade ${ }^{7}$ leading to significantly increased occurrence of deep vein thrombosis, pulmonary Emboli and acute coronary syndrome. ${ }^{9}$

This study concludes that patients with raised HbA1c contracting COVID-19 have more severe inflammation and greater lung involvement. Raised HbA1c levels were found to have positive correlation with severe radiological lung involvement, higher d-dimer levels and hence higher risk of hypercoagulability in COVID-19 patients. Higher $\mathrm{HbA1c}$ levels were also found to be associated with higher chances of requirement of oxygen therapy during hospital stay, especially in those who had more than $50 \%$ lung involvement in HRCT evaluation. Our study found no significant relation between raised HbA1c and IL-6 levels.

\section{CONCLUSION}

Based on strong association between uncontrolled HbA1c levels and severe lung involvement, higher oxygen requirement and hypercoagulability, this commonly available test can be used in covid-19 patients for early triage, proper resource allocation and optimal monitoring at the time of admission so as to guide patient management according to the risk assessment.

\section{Limitations of the study}

This is an observational study done on a small population. As the Hba1c was collected only on admission and patient follow up could not be done. Larger studies are needed to further confirm the findings based on patient follow up. Prognosis cannot be predicted based on $\mathrm{HbA1c}$.

\section{ACKNOWLEDGEMENT}

The authors take this opportunity to thank Department of Radiology, Medicine and Pathology for their whole-hearted support for this study.

\section{REFERENCES}

1. Zhenzhou W, Zhe D and Zhu Fengxue Z. Glycosylated hemoglobin is associated with systemic inflammation, hyper coagulability, and prognosis of COVID-19 patients. Diabetes Res Clin Pract. 2020; 164: 108214.

https://doi.org/10.1016/j.diabres.2020.108214

2. WHO. Coronavirus disease (COVID-19) outbreak. 2020; https:// www.who.int/emergencies/diseases/novel-coronavirus-2019 (accessed Apr 30, 2020).

3. Zhu N, Zhang D, Wang W, Li X, Yang B, Song J, et al. A novel coronavirus from patients with pneumonia in China. $\mathrm{N}$ Engl $\mathrm{J}$ Med. 2019: 382(8): 727-733.

https://doi.org/10.1056/NEJMoa2001017

4. Yogendra $M$ and Basant $P$. Relation of D-dimer levels of COVID-19 patients with diabetes mellitus. Diabetes and Metabolic Syndrome: Clinical Research and Reviews. 2020; 14 : 1927 e1930.

https://doi.org/10.1016/j.dsx.2020.09.035

5. Sudhir B and Govind R. Impact of glycemic control in diabetes mellitus on management of COVID-19 infection; International Journal of Diabetes in Developing Countries.2020; 40: 340-345. https://doi.org/10.1007/s13410-020-00868-7

6. Sebastian KJ, Dietmar F, Susanne K, Mathis S, Thomé C and Joannidis M. Unrecognized diabetes in critically ill COVID-19 patients. Critical Care. 2020; 24:406.

https://doi.org/10.1186/s13054-020-03139-3

7. Matteo A, Maria C and Michele M. COVID-19 in people with diabetes: understanding the reasons for worse outcomes; Lancet Diabetes Endocrinol. 2020: 8; 782-792. Published Online July 17, 2020.

8. Wan Z and Shandong Y. Clinical Features of COVID-19 Patients with Diabetes and Secondary Hyperglycemia; Hindawi Journal of Diabetes Research. Volume 2020, Article ID 3918723, 9 pages.

https://doi.org/10.1155/2020/3918723

9. Cooper ID, Crofts CAP, Di Nicolantonio JJ, Malhotra A, Elliot B, Kyriakidou $Y$, et al. Relationships between hyperinsulinaemia, magnesium, vitamin D, thrombosis and COVID-19: rationale for 
clinical management. Open Heart. 2020;7:e001356.

https://doi.org/10.1136/openhrt-2020-001356

10. Awadhesh S and Kamlesh K. Assessment of risk, severity, mortality, glycemic control and antidiabetic agents in patients with diabetes and COVID-19: A narrative review; international diabetes federation. Diabetes Research and Clinical Practice. 2020; 165: 108266

https://doi.org/ 10.1016/j.diabres.2020.108266

11. Ran Y, Xiang L, Huan L, Yanling Z, Xianxiang Z, Qiuxia X, et al. CT Severity Score: An Imaging Tool for Assessing Severe COVID-19. Radiology: Cardiothoracic Imaging. 2020; 2(2): e200047.

https://doi.org/10.1148/ryct.2020200047
12. World Health Organization. Laboratory testing for coronavirus disease 2019(COVID -19) In suspected human cases: interim guidance. 2020

13. International Diabetes Federation Atlas, 9th edition

14. Reference Values During Pregnancy http://www.perinatology. com/Reference/Reference\%20Range s/D-Dimer.htm) at perinatology.com. Retrieved October 2014.

15. Tobias H, Vindi J, Chiara A, Hellmuth JC, von BergweltBaildon M, Klein M, et al. Level of IL-6 predicts respiratory failure in hospitalized symptomatic COVID-19 patients.

https://doi.org/10.1101/2020.04.01.20047381; posted April 10, 2020

\section{Author's contribution:}

PG- Statistically analysed and interpreted, preparation of manuscript and revision of the manuscript; TS- Concept and design of the study; prepared first draft of manuscript; $\mathbf{A H}$ - Concept, coordination, review of literature and manuscript preparation; BKR- Interpreted the results; reviewed the literature and manuscript preparation.

\section{Work attributed to:}

L. N. Medical College and J.K. Hospital, Bhopal, MP, India.

Orcid ID:

Dr. Prakhar Gupta- (1) https://orcid.org/0000-0001-9337-4244

Dr. Tasha Samuel- (i) https://orcid.org/0000-0003-4774-4165

Dr. Ankitkumar Halani- (i) https://orcid.org/0000-0003-2334-1927

Source of Funding: None, Conflict of Interest: None. 